\title{
Coronary Calcium Score as an Adjunct to Nuclear Myocardial Perfusion Imaging for Risk Stratification Before Noncardiac Surgery
}

\author{
Jelena R. Ghadri*1, Michael Fiechter*1,2, Katja Veraguth ${ }^{1}$, Catherine Gebhard ${ }^{1}$, Aju P. Pazhenkottil ${ }^{1}$, Tobias A. Fuchs ${ }^{1}$, \\ Christian Templin ${ }^{3}$, Oliver Gaemperli*1,3, and Philipp A. Kaufmann*1,2 \\ ${ }^{I}$ Department of Radiology, Cardiac Imaging, University Hospital Zurich, Zurich, Switzerland; ${ }^{2}$ Zurich Centre for Integrative Human \\ Physiology (ZIHP), University of Zurich, Zurich, Switzerland; and ${ }^{3}$ Department of Cardiology, Cardiovascular Center, \\ University of Zurich, Zurich, Switzerland
}

The purpose of this study was to evaluate the added value of coronary artery calcium score (CACS) as an adjunct to myocardial perfusion imaging (MPI) with SPECT for cardiac risk stratification before noncardiac surgery. SPECT MPI is a well-established and widely used tool for preoperative risk stratification before noncardiac surgery. The potential added value of combining SPECT MPI with CACS is unknown. Methods: We included 326 consecutive patients who were referred for SPECT MPI for preoperative cardiac risk assessment before elective noncardiac surgery. All patients underwent an additional low-dose CT scan for CACS and SPECT MPI. Patients were followed up for $40 \mathrm{~d}$ after their index surgical procedure, and the occurrence of major adverse cardiovascular events (MACE), including death, myocardial infarction, revascularization, stroke, and sudden cardiac death, was registered. Results: Postoperative MACE occurred in 30 patients (9\%). Cumulative MACE rate was highest in patients with abnormal SPECT and high CACS $(22 \%)$, defined by a cutoff value CACS of 1,314 or more, and lowest in patients with normal SPECT MPI findings and low CACS (5\%) (CACS < 1,314). A CACS score of 1,314 or more was independently associated with a higher MACE rate in patients with normal ( $12 \%$ vs. $5 \%$ ) or abnormal perfusion $(22 \%$ vs. $12 \%, P<0.05$ for all intergroup comparisons). Conclusion: SPECT MPI findings and CACS are strong preoperative risk predictors. CACS allows further risk stratification, indicating very low risk when CACS less than 1,314 is associated with normal SPECT MPI findings. Conversely, in patients with abnormal SPECT MPI findings, a CACS of 1,314 or more confers an added value for predicting adverse outcomes.

\footnotetext{
Received Oct. 31, 2011; revision accepted Feb. 9, 2012.

For correspondence or reprints contact: Philipp A. Kaufmann, Cardiac Imaging, University Hospital Zurich, Ramistrasse 100, NUK C 42, CH-8091

Zurich, Switzerland.

E-mail: pak@usz.ch

${ }^{*}$ Contributed equally to this work.

Published online May 18, 2012.

COPYRIGHT @ 2012 by the Society of Nuclear Medicine, Inc.
}

Key Words: preoperative risk stratification; noncardiac surgery; calcium score; SPECT MPI

J Nucl Med 2012; 53:1081-1086

DOI: 10.2967/jnumed.111.100206

O plications in the aftermath of major noncardiac surgical procedures (1,2). Currently, approximately 500,000-900,000 surgical patients experience perioperative cardiac events worldwide per year (3). The increased surgical stress may contribute to acute ischemic events through different mechanisms. On one hand, the augmented metabolic demand can induce a supply-to-demand mismatch, eliciting ischemia in the presence of obstructive coronary stenoses. On the other hand, the surgical trauma leads to an activation of inflammatory and coagulation pathways $(4,5)$ predisposing to rupture and thrombosis of nonobstructive plaques. Additionally, the increasing age of the surgical population entails a higher prevalence of cardiovascular risk factors among surgical candidates.

Therefore, there is an important need for accurate risk stratification tools to identify patients at high risk for cardiovascular complications before noncardiac surgery. Clinical risk scores based on multivariate analyses of observational data have been validated as a simple method to determine cardiac perioperative risk (6,7). Moreover, noninvasive imaging techniques can be used to complement traditional risk indices. Several trials have documented the incremental prognostic value of stress echocardiography and SPECT stress myocardial perfusion imaging (MPI) before noncardiac surgery (1).

However, despite elaborate risk stratification, an impressive number of patients, estimated at 1 million per year, continues to experience cardiovascular complications around surgical procedures in the United States alone $(3,8)$. Noninvasive techniques such as stress echocardiography or SPECT MPI may detect obstructive coronary artery disease (CAD) but fail to discover subclinical atherosclerosis predisposing the patient 
to plaque rupture. The coronary artery calcium score (CACS) has been reported as a surrogate of coronary atherosclerotic burden and has been correlated with outcome in large patient cohorts (9-12). Therefore, the aim of the present study was to evaluate the added value of CACS over SPECT MPI alone for cardiac risk stratification before noncardiac surgery.

\section{MATERIALS AND METHODS}

\section{Study Design and Population}

We included consecutive patients aged $55 \mathrm{y}$ or older referred to noninvasive cardiac imaging because of suspected increased perioperative cardiac risk before elective noncardiac surgery at the University Hospital of Zurich between 2007 and 2010. The need to obtain written informed consent was waived because of the nature of the study, which included solely clinical data collection.

The prescan patient assessment consisted of a detailed interview including patient symptoms, previous cardiac events, cardiovascular risk factors, and medication. Laboratory data were obtained from medical records including serum total cholesterol, creatinine, N-terminal pro-brain natriuretic peptide, and glycated hemoglobin (HbAlc). All surgical procedures were classified into 3 categories-low $(<1 \%)$, intermediate $(1 \%-5 \%)$, or high $(>5 \%)$-for cardiovascular events as previously specified $(1,2)$. Individual surgical risk estimates were calculated on the basis of clinical parameters according to the revised index of Lee et al. (6) and graduated as classes I, II, III, and IV, representing a risk for complications of $0.4 \%, 0.9 \%, 6.6 \%$, and $11.0 \%$, respectively.

\section{SPECT MPI and CT Protocols}

All patients underwent 1-d stress-rest SPECT MPI. Standard pharmacologic stress was induced with adenosine infusion (140 $\mu \mathrm{g} / \mathrm{kg} / \mathrm{min}$ ) or dobutamine infusion ( $\leq 40 \mu \mathrm{g} / \mathrm{kg} / \mathrm{min}$ ). A dose of $300-350 \mathrm{MBq}$ of ${ }^{99 \mathrm{~m}} \mathrm{Tc}$-tetrofosmin for stress SPECT MPI and a 3-fold higher dose for rest SPECT MPI were administered (13). SPECT MPI studies were performed on a dual-head camera (Infinia or Ventri; GE Healthcare) and reconstructed on a dedicated workstation (Xeleris; GE Healthcare) using an iterative reconstruction algorithm with CT-based attenuation correction, as previously reported (14).

CT images were acquired on a LightSpeed VCT 64-slice standalone CT scanner (GE Healthcare). The same low-dose, unenhanced CT scan was used for SPECT MPI attenuation correction and CACS as previously reported (15). The scanning parameters were as follows: prospective electrocardiogram triggering, 2.5$\mathrm{mm}$ slice thickness, $120 \mathrm{-kV}$ tube voltage, $200 \mathrm{mAs}$ per rotation tube current, and a $50 \times 50 \mathrm{~cm}$ large scan field of view (16). The coregistration between SPECT and CT images (for attenuation correction) was accomplished by visual quality control of emission scans (SPECT) and transmission scans (CT) using commercially available fusion software, as shown elsewhere (15). CT images were transferred to a dedicated workstation for CACS (Advantage workstation 4.2 or 4.4; GE Healthcare).

\section{SPECT Myocardial Perfusion and CT Image Interpretation}

SPECT myocardial perfusion images were visually analyzed by the consensus of 2 independent observers, and segmental tracer uptake was evaluated using a semiquantitative scoring system, as recommended by the American Society of Nuclear Cardiology (17). A summed stress score of 4 or greater was considered ab- normal, and a summed difference score of 2 or greater represented clinically relevant ischemia $(18,19)$.

CACS were calculated by an experienced operator using commercially available software (Smartscore 4.0; GE Healthcare) according to Agatston et al. (20). Lesions were manually traced on CT images, and vessel-specific scores were added to yield the total CACS (16).

\section{Follow-up and Outcomes}

Patients were followed up for $40 \mathrm{~d}$ after surgery. The study endpoint was defined as a composite of major adverse cardiovascular events (MACE) including death, nonfatal myocardial infarction (MI), urgent revascularization, stroke, and aborted sudden cardiac death. MI was defined according to Thygesen et al. (21). Stroke was considered as an endpoint if reviewed independently by a neurologist and classified on the basis of a focal neurologic deficit lasting $24 \mathrm{~h}$ or more, with a clinically relevant lesion on brain imaging and no nonvascular cause.

\section{Statistical Analysis}

Statistical analysis was performed with SPSS software (version 19.0; SPSS Inc.). Numeric data are given as mean \pm SD or median with interquartile range (IQR) when appropriate and categoric data as frequencies (percentages). For numeric data, normal distribution was tested using quantile-quantile plots and confirmed using the Shapiro-Wilk test. Intergroup comparison of numeric data was performed with the Mann-Whitney $U$ test, whereas categoric data were compared with the $\chi^{2}$ test or Fisher exact test.

CACS and SPECT findings were correlated using binary logistic regression analysis, and results were expressed as odds ratio, with its respective 95\% confidence interval (CI). Receiver-operatingcharacteristic analysis was applied to detect the optimal CACS cutoff to predict postoperative events and the predictive power estimated on the basis of the area under the receiver-operatingcharacteristic curve. Differences in survival over time were analyzed by the Kaplan-Meier method and compared using the $\log$-rank test. Univariate and multivariate Cox proportional hazards regression models were used to identify independent predictors of MACE. Variables included in the model were selected in a stepwise forward-selection manner. Entry and retention sets with a $P$ value of less than 0.05 indicated a significant difference. A variable's risk was expressed as hazard ratio (HR), with corresponding 95\% CI. A $P$ value of less than 0.05 was considered statistically significant.

\section{RESULTS \\ Patient Characteristics}

After the exclusion of patients because of lack of consent, missing follow-up, devices potentially causing artifacts, or rescheduled surgical intervention beyond the predefined 100-d window, the 326 remaining patients were included into the final analysis.

The mean age of the study cohort was $71.1 \pm 8.5 \mathrm{y}$, and 79 (24\%) patients were women and 247 (76\%) men (Table 1).

\section{Type of Surgery and Clinical Risk Estimates}

Surgical risk was classified as low, intermediate, and high in $40(12 \%), 192(59 \%)$, and 94 (29\%) patients. Clinical risk estimates for perioperative cardiovascular complications based on the revised Lee index (6) were distributed as follows: class I, 90 (28\%) patients; class II, 154 (47\%) patients; class III, 61 (19\%) patients; and class IV, 21 (6\%) patients. 
TABLE 1

Baseline Characteristics $(n=326)$

\begin{tabular}{|c|c|}
\hline Characteristic & Value \\
\hline Mean age $\pm S D(y)$ & $71.1 \pm 8.5$ \\
\hline \multicolumn{2}{|l|}{$\operatorname{Sex}(n)$} \\
\hline Male & $247(76)$ \\
\hline Female & $79(24)$ \\
\hline Mean body mass index $\pm \mathrm{SD}\left(\mathrm{kg} / \mathrm{m}^{2}\right)$ & $26 \pm 6$ \\
\hline \multicolumn{2}{|l|}{ Cardiovascular risk factors $(n)$} \\
\hline Arterial hypertension & $235(72)$ \\
\hline Dyslipidaemia & $218(67)$ \\
\hline Current or former smoker & $219(67)$ \\
\hline Diabetes mellitus & $68(21)$ \\
\hline Positive family history & $31(10)$ \\
\hline \multicolumn{2}{|l|}{ Cardiovascular history $(n)$} \\
\hline Known CAD & $132(41)$ \\
\hline Revascularization & $97(30)$ \\
\hline Myocardial infarction & $62(19)$ \\
\hline Stroke or transient ischemic attack & $39(12)$ \\
\hline Congestive heart failure & $26(8)$ \\
\hline \multicolumn{2}{|l|}{ Medication (n) } \\
\hline Aspirin & $201(62)$ \\
\hline $\begin{array}{l}\text { Angiotensin-converting enzyme inhibitor } \\
\text { or angiotensin-receptor blocker }\end{array}$ & $181(56)$ \\
\hline$\beta$-blocker & $167(51)$ \\
\hline Statin & $178(55)$ \\
\hline Nitroglycerin & $14(4)$ \\
\hline
\end{tabular}

\section{SPECT MPI and CACS Findings}

SPECT MPI perfusion was normal in $242(74 \%)$ patients and abnormal in $84(26 \%)$ patients. Twenty patients $(6 \%)$ had a reversible defect, 47 (14\%) had a fixed defect, and 17 $(5 \%)$ a partially reversible defect.

Patients with known CAD had a significantly higher CACS (mean, 110 vs. $631 ; P<0.001$ ) and were more likely to have SPECT abnormalities $(51 \%, 67 / 132$, vs. 9\%, 17/194; $P<$ 0.001). The median CACS was 759 (IQR, 150-1,683). A CACS of zero was found in $16(5 \%)$ patients, $1-99$ in 53 (16\%), 100-399 in $54(17 \%), 400-1,000$ in $67(21 \%)$, and greater than 1,000 in $136(42 \%)$. Figure 1 shows the distribution of CACS in relation to SPECT MPI results in the total study cohort. A stepwise increase in the proportion of pathologic SPECT MPI findings could be observed with increasing CACS $(P=0.003)$. There was a significant correlation between the logarithmic CACS and the likelihood of an abnormal SPECT result (odds ratio, 1.7; 95\% CI, 1.2-2.4; $P=0.002$ ). The estimated radiation dose was $0.9 \pm 0.1 \mathrm{mSv}$ for CACS scanning and $9.47 \pm 1.3 \mathrm{mSv}$ for SPECT MPI scanning.

\section{Follow-up Data}

The follow-up of $40 \mathrm{~d}$ was complete for all 326 patients included in the analysis and yielded a total of $30(9 \%)$ MACE: 15 deaths, 3 nonfatal MIs, 2 aborted sudden cardiac deaths, 3 strokes, and 7 revascularizations. A MACE rate in patients with CACS above and below the threshold of 1,314 in relation to SPECT MPI findings is given in Table 2.
The MACE rates in relation to the CACS was as follows: $0 \%$ for a CACS of 0 (IQR, $0-0$ ), 3.8\% for a CACS of 1-99 (IQR, 12-68), 11.1\% for a CACS of 100-399 (IQR, 151.25285.5), 7.5\% for a CACS of 400-999 (IQR, 501.5-838.5), and $12.5 \%$ for a CACS or more than 1,000 (IQR, 1,4442,660).

Receiver-operating-characteristic analysis resulted in an optimal CACS threshold of 1,314 (area under the receiveroperating-characteristic curve, $0.64 ; 95 \% \mathrm{CI}, 0.54-0.73$; $P=0.013)$ to predict postoperative events. Figure 2 shows Kaplan-Meier survival curves for cumulative postoperative survival free of MACE, stratified according to SPECT MPI results and high $(\geq 1,314)$ or low $(<1,314)$ CACS. The cumulative MACE rate was highest in patients with abnormal SPECT and high CACS (22\%) and lowest in patients with normal SPECT MPI findings and low CACS (5\%). In patients with normal SPECT MPI findings but high CACS, or in patients with abnormal SPECT MPI findings but low CACS, the risk was intermediate (12\%) (Fig. 3).

On univariate analysis, the following parameters emerged as predictors of MACE (Table 3): Lee index, abnormal SPECT MPI findings, logarithmic CACS, and a CACS of 1,314 or greater. After adjustment for parameters entered into the multivariate model, the Lee index (HR, $1.55 ; 95 \% \mathrm{CI}$, $1.06-2.26 ; P=0.022$ ) and a CACS of 1,314 or greater (HR, 2.21; 95\% CI, 1.04-4.67; $P=0.038$ ) were identified as significant independent predictors.

\section{DISCUSSION}

The results of the present study demonstrate an added prognostic predictive value of CACS over SPECT MPI findings alone because a CACS of 1,314 or greater was an independent predictor of adverse outcome. In patients with normal perfusion by SPECT MPI, a CACS of 1,314 or

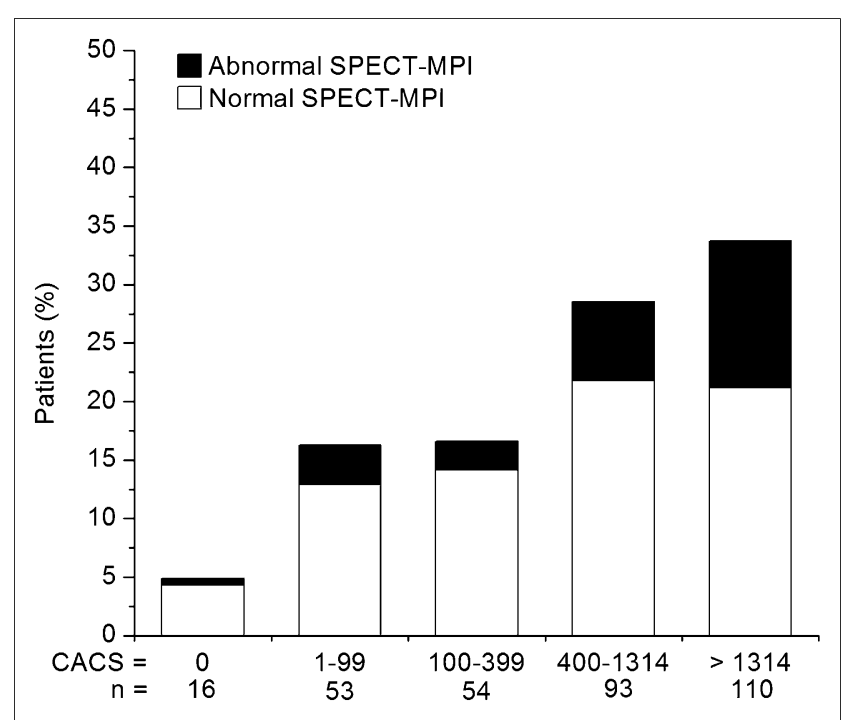

FIGURE 1. Bar graph showing prevalence of normal or abnormal SPECT MPI findings across subgroups of CACS. $y$-axis denotes percentage of patients taken from entire study population of 326 patients. 


\begin{tabular}{ll}
\hline \multicolumn{1}{c}{ Parameter } & No. of MACE patients (of 30 total) \\
\hline Separate predictive values of SPECT MPI and CACS & \\
SPECT MPI & $16(7)$ \\
$\quad$ Normal findings $(n=242)$ & $14(17)$ \\
Abnormal findings $(n=84)$ & $13(6)$ \\
CACS & $17(16)$ \\
$\quad<1,314(n=216)$ & \\
$\geq 1,314(n=110)$ & \\
Added value of CACS combined with SPECT MPI & $8(5)$ \\
Normal SPECT MPI findings & $8(12)$ \\
$\quad<1,314(n=173)$ & \\
$\geq 1,314(n=69)$ & $5(12)$ \\
Abnormal SPECT MPI findings & $9(22)$ \\
$<1,314(n=43)$ & 0.005 \\
$\geq 1,314(n=41)$ & 0.002
\end{tabular}

$P$ values indicate results from log-rank test. Data in parentheses are percentages.

greater allowed further discrimination of patients with a high MACE rate versus those with a low rate within $40 \mathrm{~d}$ from the surgical procedure. Thus, despite normal perfusion, patients with a CACS of 1,314 or greater are at increased risk for cardiovascular events. In these patients, the massive coronary calcification probably reflects concomitant endothelial and microvasculatory dysfunction. This is in line with recent studies documenting that an increased CACS is a predictor of adverse outcome in patients with normal MPI findings using ${ }^{82} \mathrm{Rb}$ and PET (22) or ${ }^{99 \mathrm{~m}} \mathrm{Tc}$-tetrofosmin and SPECT (15). In addition, in a prospective study, a very high CACS has been shown to unmask obstructive CAD in patients with normal SPECT MPI findings (23). Our study extends this observation to preoperative risk assessment. Whether the clinical implementation of this tool translates into improved risk modification and prognostic benefit remains to be elucidated. Interestingly, even in patients with documented perfusion abnormalities, CACS proved to be an important prognostic indicator, with a strong incremental predictive value. This may be at least in part attributable to the fact that a very high CACS may indicate extension of the disease beyond the epicardial section of the coronary tree down to the microcirculation, including endothelial dysfunction. Thus, a very high CACS represents a more deteriorated stage of $\mathrm{CAD}$, which may confer a higher risk of adverse events. Although CACS has been well established as a long-term predictor of disease progression and adverse events $(22,24,25)$, the present study is the first, to our knowledge, to document a role for CACS in short-term preoperative risk stratification before noncardiac surgery. In fact, whereas in several studies the impact of myocardial ischemia as assessed by stress echocardiography or SPECT MPI has been evaluated (2), no data are available on the role of CACS in this setting.

Several noninvasive imaging techniques have been proposed for cardiovascular risk stratification before noncardiac surgery; however, the understanding of the pathophysiology of perioperative ischemic cardiovascular is still not clear. Several mechanisms are involved in the pathogenesis of acute ischemic events. On one hand, a substantial proportion of ischemic events may be triggered by a perioperative increase in myocardial oxygen demand in the setting of a fixed highgrade coronary stenosis (26). On the other hand, the surgical stress gives rise to inflammatory and hypercoagulable states that trigger plaque rupture or erosion with subsequent vessel thrombosis (3). The latter mechanism accounts for approximately $50 \%$ of acute coronary events in the perioperative setting (1), and several studies suggest that a substantial fraction of plaque rupture occurred at sites of mild stenosis on preoperative angiographic assessment (27).

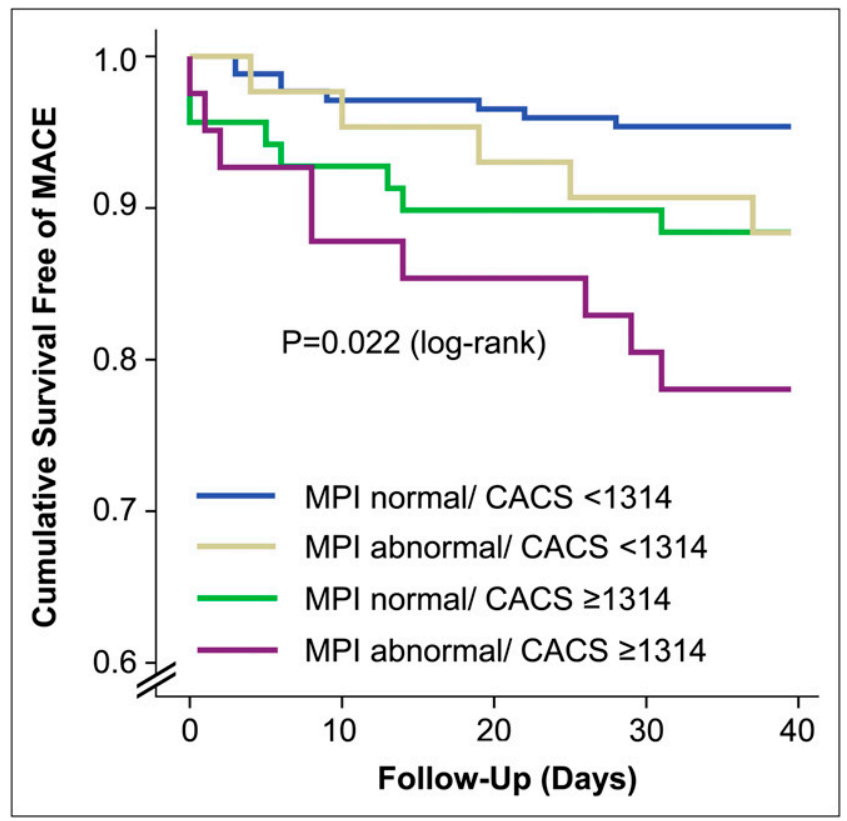

FIGURE 2. Kaplan-Meier survival curves for cumulative survival free of MACE according to subgroups of normal or abnormal SPECT MPI findings and CACS above or below $1,314(P<0.05$, log-rank test). 


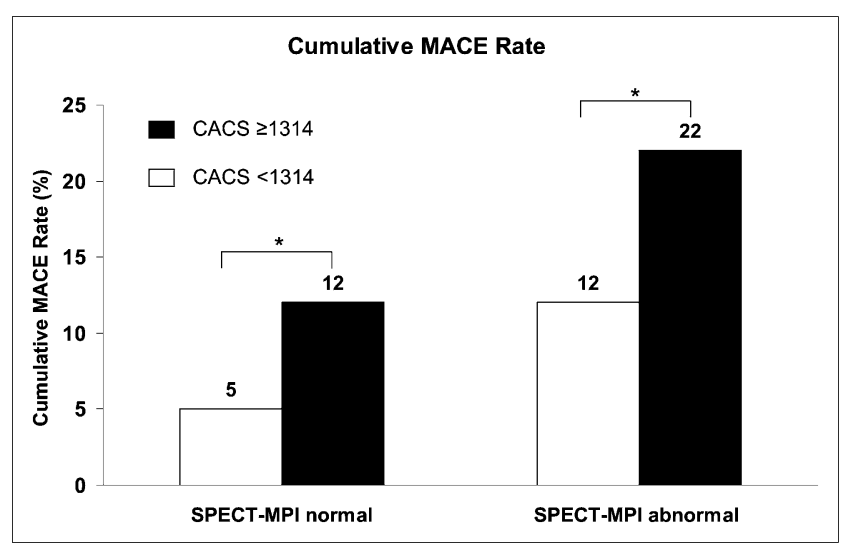

FIGURE 3. Cumulative MACE rate for patients with normal or abnormal SPECT MPI findings and CACS above or below cutoff value of $1,314(P<0.05$, log-rank test).

However, MPI or stress echocardiography can detect only obstructive coronary lesions inducing myocardial ischemia and therefore may fail to identify patients with subclinical atherosclerosis at substantial risk for perioperative plaque rupture. CACS, on the other hand, provides an estimate of overall coronary atherosclerotic burden regardless of the presence or absence of obstructive lesions. Thus, an integrated approach of functional information on myocardial perfusion by SPECT MPI and morphologic information on coronary atherosclerotic burden by CACS may provide a comprehensive picture of coronary risk and improve preoperative risk stratification. This paradigm is supported by the findings of the present study, showing an incremental value of CACS for predicting cardiovascular complications after noncardiac surgery. Interestingly, the risk evaluation tools such as preoperative risk and Lee index may be of clinical value but are at least in part discordant. In fact, the Lee index classified $75 \%$ of our study population as low risk (classes I and II) whereas the surgical risk classified $88 \%$ as intermediate and high. Although some authors have suggested systematic invasive coronary angiography in all patients before major vascular surgery (28), the incremental value of CACS may help further in shifting the tip of the balance in favor of noninvasive imaging, to avoid unnecessary preoperative invasive coronary angiography.

A limitation of the current study is that its observational nature does not allow conclusions on the impact of the added predictive value by CACS on outcome to be drawn. Determining the impact would require randomization of patients to different treatment strategies such as revascularization versus optimal medical treatment, with a prospective interventional trial design, which was beyond the scope of the present study.

\section{CONCLUSION}

SPECT MPI findings and CACS are strong preoperative risk predictors. CACS allows further risk stratification, indicating very low risk when a CACS less than 1,314 is associated with normal SPECT MPI findings. Conversely, in patients with abnormal SPECT MPI findings a CACS of 1,314 or greater confers an added value for predicting adverse outcome. In addition, our findings may not necessarily be extrapolated to a population not undergoing surgery because Figure 2 illustrates that an important part of the added risk in patients with high CACS was posed by the surgical procedures and not by the natural course of the disease. Finally, we have limited our observations to patients aged 55 y or older, because elderly patients require surgery 4 times more often than younger populations and because the surgical risk of MACE is particularly relevant in patients at risk for $\mathrm{CAD}$, which starts rising after this age (1).

\section{DISCLOSURE STATEMENT}

The costs of publication of this article were defrayed in part by the payment of page charges. Therefore, and solely to indicate this fact, this article is hereby marked "advertisement" in accordance with 18 USC section 1734.

\section{ACKNOWLEDGMENTS}

We thank Ennio Müller, Edlira Loga, and Serpil BostanciKökyildrim for their excellent technical support. The study was supported by a grant from the Swiss National Science

TABLE 3

Predictor of MACE on Cox Proportional Hazards Regression Models

\begin{tabular}{|c|c|c|c|c|c|c|}
\hline \multirow[b]{2}{*}{ Characteristic } & \multicolumn{3}{|c|}{ Univariate } & \multicolumn{3}{|c|}{ Multivariate } \\
\hline & HR & $95 \% \mathrm{Cl}$ & $P$ & $\mathrm{HR}$ & $95 \% \mathrm{Cl}$ & $P$ \\
\hline Age & 1.03 & $0.99-1.07$ & 0.141 & NA & & NS \\
\hline Sex & 0.79 & $0.32-1.929$ & 0.58 & NA & & NS \\
\hline Surgical risk estimate & 1.32 & $0.73-2.37$ & 0.34 & NA & & NS \\
\hline Lee index & 1.73 & $1.20-2.46$ & 0.003 & 1.55 & $1.06-2.26$ & 0.022 \\
\hline SPECT MPI & 2.61 & $1.28-5.36$ & 0.009 & NA & & NS \\
\hline CACS log & 2.10 & $1.15-3.83$ & 0.015 & NA & & NS \\
\hline $\mathrm{CACS} \geq 1,314$ & 2.72 & $1.32-5.60$ & 0.007 & 2.2 & $1.04-4.67$ & 0.038 \\
\hline
\end{tabular}


Foundation (SNSF) and by the ZHIP (Zurich Center for Integrative Human Physiology, University of Zurich, Switzerland). No other potential conflict of interest relevant to this article was reported.

\section{REFERENCES}

1. Poldermans D, Bax JJ, Boersma E, et al. Guidelines for pre-operative cardiac risk assessment and perioperative cardiac management in non-cardiac surgery. Eur Heart J. 2009;30:2769-2812.

2. Fleisher LA, Beckman JA, Brown KA, et al. ACC/AHA 2007 guidelines on perioperative cardiovascular evaluation and care for noncardiac surgery: a report of the American College of Cardiology/American Heart Association Task Force on Practice Guidelines (Writing Committee to Revise the 2002 Guidelines on Perioperative Cardiovascular Evaluation for Noncardiac Surgery) developed in collaboration with the American Society of Echocardiography, American Society of Nuclear Cardiology, Heart Rhythm Society, Society of Cardiovascular Anesthesiologists, Society for Cardiovascular Angiography and Interventions, Society for Vascular Medicine and Biology, and Society for Vascular Surgery. J Am Coll Cardiol. 2007;50:e159-e241.

3. Devereaux PJ, Goldman L, Cook DJ, Gilbert K, Leslie K, Guyatt GH. Perioperative cardiac events in patients undergoing noncardiac surgery: a review of the magnitude of the problem, the pathophysiology of the events and methods to estimate and communicate risk. CMAJ. 2005;173:627-634.

4. Flinn WR, McDaniel MD, Yao JS, Fahey VA, Green D. Antithrombin III deficiency as a reflection of dynamic protein metabolism in patients undergoing vascular reconstruction. J Vasc Surg. 1984;1:888-895.

5. McDaniel MD, Pearce WH, Yao JS, et al. Sequential changes in coagulation and platelet function following femorotibial bypass. J Vasc Surg. 1984;1:261-268.

6. Lee TH, Marcantonio ER, Mangione CM, et al. Derivation and prospective validation of a simple index for prediction of cardiac risk of major noncardiac surgery. Circulation. 1999;100:1043-1049.

7. Boersma E, Kertai MD, Schouten O, et al. Perioperative cardiovascular mortality in noncardiac surgery: validation of the Lee cardiac risk index. Am J Med. 2005; 118:1134-1141.

8. Mangano DT, Goldman L. Preoperative assessment of patients with known or suspected coronary disease. N Engl J Med. 1995;333:1750-1756.

9. Shaw LJ, Raggi P, Schisterman E, Berman DS, Callister TQ. Prognostic value of cardiac risk factors and coronary artery calcium screening for all-cause mortality. Radiology. 2003;228:826-833.

10. Budoff MJ, Shaw LJ, Liu ST, et al. Long-term prognosis associated with coronary calcification: observations from a registry of 25,253 patients. J Am Coll Cardiol. 2007;49:1860-1870.

11. Detrano R, Guerci AD, Carr JJ, et al. Coronary calcium as a predictor of coronary events in four racial or ethnic groups. N Engl J Med. 2008;358:1336-1345.

12. Raggi $\mathrm{P}$, Callister TQ, Cooil B, et al. Identification of patients at increased risk of first unheralded acute myocardial infarction by electron-beam computed tomography. Circulation. 2000;101:850-855.

13. Hesse B, Tagil K, Cuocolo A, et al. EANM/ESC procedural guidelines for myocardial perfusion imaging in nuclear cardiology. Eur J Nucl Med Mol Imaging. 2005;32:855-897.
14. Valenta I, Treyer V, Husmann L, et al. New reconstruction algorithm allows shortened acquisition time for myocardial perfusion SPECT. Eur J Nucl Med Mol Imaging. 2010;37:750-757.

15. Schepis T, Gaemperli O, Koepfli P, et al. Use of coronary calcium score scans from stand-alone multislice computed tomography for attenuation correction of myocardial perfusion SPECT. Eur J Nucl Med Mol Imaging. 2007;34: 11-19.

16. Ghadri JR, Goetti R, Fiechter M, et al. Inter-scan variability of coronary artery calcium scoring assessed on 64-multidetector computed tomography vs. dualsource computed tomography: a head-to-head comparison. Eur Heart J. 2011;32: 1865-1874.

17. Holly TA, Abbott BG, Al-Mallah M, et al. Single photon-emission computed tomography. J Nucl Cardiol. 2010;17:941-973.

18. Hachamovitch R, Berman DS, Shaw LJ, et al. Incremental prognostic value of myocardial perfusion single photon emission computed tomography for the prediction of cardiac death: differential stratification for risk of cardiac death and myocardial infarction. Circulation. 1998;97:535-543.

19. Sharir T, Germano G, Kang X, et al. Prediction of myocardial infarction versus cardiac death by gated myocardial perfusion SPECT: risk stratification by the amount of stress-induced ischemia and the poststress ejection fraction. $J$ Nucl Med. 2001;42:831-837.

20. Agatston AS, Janowitz WR, Hildner FJ, Zusmer NR, Viamonte M, Detrano R. Quantification of Coronary-Artery Calcium Using Ultrafast Computed-Tomography. J Am Coll Cardiol. 1990;15:827-832.

21. Thygesen K, Alpert JS, White HD. Universal definition of myocardial infarction. Circulation. 2007;116:2634-2653.

22. Schenker MP, Dorbala S, Hong EC, et al. Interrelation of coronary calcification, myocardial ischemia, and outcomes in patients with intermediate likelihood of coronary artery disease: a combined positron emission tomography/computed tomography study. Circulation. 2008;117:1693-1700.

23. Ghadri JR, Pazhenkottil AP, Nkoulou RN, et al. Very high coronary calcium score unmasks obstructive coronary artery disease in patients with normal SPECT MPI. Heart. 2011;97:998-1003.

24. Chang SM, Nabi F, Xu J, et al. The coronary artery calcium score and stress myocardial perfusion imaging provide independent and complementary prediction of cardiac risk. J Am Coll Cardiol. 2009;54:1872-1882.

25. van Werkhoven JM, Schuijf JD, Gaemperli O, et al. Incremental prognostic value of multi-slice computed tomography coronary angiography over coronary artery calcium scoring in patients with suspected coronary artery disease. Eur Heart J. 2009;30:2622-2629.

26. Dawood MM, Gutpa DK, Southern J, Walia A, Atkinson JB, Eagle KA. Pathology of fatal perioperative myocardial infarction: implications regarding pathophysiology and prevention. Int J Cardiol. 1996;57:37-44.

27. Cohen MC, Aretz TH. Histological analysis of coronary artery lesions in fatal postoperative myocardial infarction. Cardiovasc Pathol. 1999;8:133-139.

28. Monaco M, Stassano P, Di Tommaso L, et al. Systematic strategy of prophylactic coronary angiography improves long-term outcome after major vascular surgery in medium- to high-risk patients: a prospective, randomized study. $J$ Am Coll Cardiol. 2009;54:989-996. 